\title{
Uso de Topotecan no Tratamento do Tumor Epitelial de Ovário Refratário à Platina: Experiência do INCA
}

\author{
Topotecan for Treatment of Platinum-Resistant Epithelial Ovarian Cancer: the \\ Experience at the Brazilian National Cancer Institute
}

\author{
Luiz Henrique de Lima Araújo', Fernando Meton de Alencar Câmara Vieira², Isabele Ávila Small², Paulo Alexandre Ribeiro Mora³, \\ Luciana Camillo Coura ${ }^{3}$, Gustavo Henrique Gomes Advíncula
}

\section{Resumo}

Introdução: $\mathrm{O}$ câncer de ovário é a principal causa de morte entre os tumores malignos ginecológicos nos Estados Unidos. Apesar dos avanços da terapia inicial, a maioria das pacientes apresentará recaída e necessitará de tratamento adicional. Topotecan é um agente quimioterápico estabelecido para o tratamento de câncer de ovário refratário à platina e foi utilizado como droga de escolha no Instituto Nacional de Câncer (INCA) para essas pacientes. O presente estudo tem como objetivo descrever estes casos. Metodologia: Estudo de seguimento, retrospectivo e descritivo realizado através de revisão dos prontuários das pacientes, com diagnóstico de câncer epitelial de ovário refratário à platina, que receberam tratamento com topotecan no INCA, no período de janeiro de 2003 a dezembro de 2005. Resultados: Trinta e uma pacientes preencheram critérios para inclusão no estudo. Com seguimento mediano de 12 meses (3-36), o tempo mediano livre de progressão foi de quatro meses (IC 95\%; 2,1-5,8) e a mediana estimada de sobrevida global foi de 14 meses (IC 95\%; 5,1-22,8). A progressão locorregional foi a mais comum, descrita em 22 (81,5\%). Foram encontradas: taxas de resposta clínica de 22,6\%, resposta bioquímica de $25,8 \%$ e resposta radiológica de 20\%. Conclusão: O presente estudo evidencia o benefício do tratamento com topotecan na população estudada, com taxa de resposta, tempo livre de progressão e sobrevida global, que estão de acordo com os da literatura.

Palavras-chave: Neoplasias ovarianas; Quimioterapia; Topotecan

${ }^{1}$ Médico-residente de Oncologia Clínica - Instituto Nacional de Câncer (INCA)

${ }^{2}$ Pesquisador do Serviço de Pesquisa Clínica / INCA

${ }^{3}$ Preceptor de Oncologia Clínica / INCA

Departamento de Oncologia Clínica do Hospital de Câncer II / INCA

Endereço para correspondência: Luiz Henrique de Lima Araújo. Rua Washington Luis, 85 / 601 - Centro - Rio de Janeiro (RJ), Brasil.

E-mail: luizmed@uol.com.br 


\section{INTRODUÇÃO}

O câncer de ovário é a principal causa de morte entre os tumores malignos ginecológicos nos Estados Unidos ${ }^{1}$, sendo o oitavo em incidência e quinto maior causador de morte entre mulheres com tumores malignos, excluindo aqueles de pele não melanoma ${ }^{1}$. Para 2007, a Sociedade Americana de Câncer estimou 22.430 casos novos e 15.280 mortes por câncer de ovário ${ }^{1}$. Nos últimos 25 anos, a sobrevida em cinco anos teve modesta melhora, de 37\% no início da década de 1970 para 44\% no ano $2000 .{ }^{1}$ No Brasil, a incidência de câncer de ovário ajustada por idade descrita na cidade de Goiânia foi de 6,71 casos por 100.000 habitantes entre os anos de 1996 e 2000 , o que corresponde a $1,77 \%$ do total de casos de câncer, excluindo o câncer in situ da cérvix uterina e outras neoplasias malignas da pele ${ }^{2}$.

O câncer epitelial é o principal tipo histológico e se apresenta comumente em estádios avançados ${ }^{3}$. Aproximadamente, $75 \%$ das mulheres com câncer epitelial de ovário se encontram em estágio III ou IV ao diagnóstico ${ }^{3}$. Cirurgia agressiva com intuito de remover toda a doença macroscópica, ou seja, a cirurgia de citorredução, é parte integral do tratamento inicial do câncer de ovário avançado ${ }^{4}$. Os benéficos teóricos desta abordagem incluem ressecção de grandes tumores necróticos com pouco suprimento sangüíneo e de grandes tumores, que se encontram em fase lenta de proliferação, deixando apenas doença residual mínima ou doença microscópica, com maior potencial de sensibilidade à quimioterapia ${ }^{4}$. Existem diferentes conceituações para a citorredução ótima, porém a ressecção de toda a doença macroscópica ou doença residual menor do que um centímetro é a mais amplamente aceita ${ }^{4}$. Há evidência de que o tamanho de doença residual tenha relação inversa com sobrevida livre de progressão e sobrevida global ${ }^{4}$. Infelizmente, apesar dos avanços da terapia atual, incluindo citorredução agressiva e tratamento adjuvante com quimioterapia baseada em platina e taxanos, a maioria das pacientes vai apresentar recaída e eventualmente morrer da doença ${ }^{3}$. Um número relativamente pequeno dessas pacientes com doença recorrente pode ser curado. Múltiplos esquemas terapêuticos podem ser oferecidos com intuito paliativo, melhorando a qualidade de vida e sobrevida livre de progressão ${ }^{3}$. A terapia de resgate é comumente baseada na sensibilidade ao tratamento com platina $^{5}$. São consideradas refratárias à platina aquelas pacientes que apresentam progressão da doença durante o tratamento ou que têm intervalo livre de doença menor do que seis meses após o tratamento primário com platina $^{5}$. Essas pacientes geralmente têm um pior prognóstico e o tratamento com a mesma combinação inicial de quimioterápicos resulta em toxicidade significativa, sem benefício de sobrevida ${ }^{3}$. Diversas opçôes terapêuticas podem ser usadas no resgate dessas pacientes, dentre as quais estão topotecan, doxorrubicina lipossomal, etoposídeo e gencitabina, com resposta global média variando de $20 \%$ a $40 \%{ }^{4}$.

O tratamento paliativo em pacientes com tumor epitelial de ovário recorrente pode também incluir a radioterapia ${ }^{4}$. Sintomas oriundos de massa pélvica em crescimento, incluindo dor, sangramento e constipação intestinal secundária à estenose retal podem ser controlados com radioterapia pélvica, reduzindo o uso de analgésicos e evitando a necessidade de colostomia. Doença metastática para pulmão, ossos e sistema nervoso central também pode ser tratada paliativamente com radioterapia ${ }^{4}$.

\section{JUSTIFICATIVA}

Topotecan, um inibidor da topoisomerase-1, é um agente quimioterápico estabelecido para o tratamento de segunda-linha em pacientes com câncer de ovário ${ }^{4-10}$. Sua eficácia e perfil de toxicidade foram amplamente analisados em estudos de fase II publicados a partir de 1997, envolvendo principalmente pacientes refratárias à quimioterapia de primeira-linha baseada em platina ${ }^{6-10}$. A dose inicial utilizada em todos esses estudos foi de 1,5 $\mathrm{mg} / \mathrm{m}^{2} / \mathrm{dia}$, em cinco dias consecutivos, administrada em infusão de 30 minutos, repetida em intervalos de 21 dias. Foram observadas taxa de resposta global e de doença estável, variando de $13,7 \%$ a $32,6 \%$ e $27,3 \%$ a $60 \%{ }^{6-10}$. O tempo mediano de resposta documentada variou de 18,1 semanas a 11,2 meses $^{6-10}$. O intervalo mediano livre de progressão foi de quatro a 9,6 meses em pacientes com doença estável e 7,5 a 13,2 meses em pacientes, que responderam ao topotecan ${ }^{6-10}$. A sobrevida mediana variou de 47 semanas até 20,2 meses $^{6-10}$. A principal toxicidade associada ao topotecan foi a mielossupressão, porém não cumulativa. Neutropenia graus 3 e 4 foi observada em $69,1 \%$ a $100 \%$ dos pacientes, enquanto neutropenia febril ocorreu em 4,3\% a $41,7 \% \%^{6-10}$. A incidência de trombocitopenia variou de $30 \%$ a $66,7 \% \%^{6-10}$. Efeitos tóxicos não-hematológicos, como náuseas, vômitos e fadiga, foram comumente leves e raros, ocorrendo em menos de $10 \%$ dos pacientes ${ }^{6-10}$. Entre os pacientes refratários à platina, observou-se taxa de resposta global, variando de 5,9\% a $14 \%^{6-10}$.

Estudos de fase III vieram a consolidar o uso do topotecan. Ten Bokkel et al. compararam esta droga com paclitaxel em um estudo holandês randomizado, multicêntrico, envolvendo 226 pacientes com carcinoma 
epitelial avançado de ovário, que progrediram doença durante ou após terapia baseada em platina ${ }^{11}$. As pacientes foram submetidas à randomização para receber topotecan, na dose de $1,5 \mathrm{mg} / \mathrm{m}^{2} / \mathrm{dia}$, por cinco dias consecutivos, em infusão de 30 minutos, a cada 21 dias ou paclitaxel, $175 \mathrm{mg} / \mathrm{m}^{2}$ em infusão de três horas a cada 21 dias. As pacientes refratárias à platina tiveram taxa de resposta global de $13,3 \%$ e $6,7 \%$ com topotecan e paclitaxel, respectivamente $(\mathrm{p}=0,303)$. Aquelas sensíveis à platina tratadas com topotecan e paclitaxel também tiveram respostas semelhantes, de $28,8 \%$ e $20 \%$, respectivamente $(\mathrm{p}=0,213) . \mathrm{Na}$ análise final, a taxa de resposta global foi de $20,5 \%$ e $13,2 \%$ para as pacientes tratadas com topotecan e paclitaxel, respectivamente $(\mathrm{p}=0,138)$. A duração mediana de resposta foi de $32 \mathrm{e}$ 20 semanas para topotecan e paclitaxel, respectivamente $(\mathrm{p}=0,222)$ e o tempo médio para progressão de doença foi de 23 e 14 semanas, respectivamente $(\mathrm{p}=0,002)$. A sobrevida mediana das pacientes tratadas com topotecan foi de 61 semanas, comparada a 43 semanas no grupo de paclitaxel $(p=0,515)$. Neutropenia foi significativamente mais freqüente no grupo do topotecan (79\%), comparado a $23 \%$ no grupo do paclitaxel $(p<0,01)$. O estudo concluiu que topotecan tem eficácia pelo menos equivalente à do paclitaxel $^{11}$.

Em análise publicada posteriormente, Gore et al. estudaram 110 pacientes, que cruzaram de grupo e receberam terapia de terceira-linha, após progredir doença, uma vez tendo recebido terapia de segundalinha com topotecan ou paclitaxel. Foram incluídas pacientes de vários centros, envolvendo Estados Unidos e países da Europa ${ }^{12}$.

A taxa de resposta foi de $13 \%$ para o grupo que recebeu topotecan como terceira-linha, e de $10 \%$ para o grupo do paclitaxel $(\mathrm{p}=0,638)$. A duração mediana de resposta foi de 29 semanas com topotecan e de 27 semanas com paclitaxel. O tempo mediano para progressão foi de nove semanas nos dois grupos, e a sobrevida global mediana foi de 40 e 48 semanas para os grupos do topotecan e paclitaxel, respectivamente. Não houve diferença estatisticamente significativa. Também, foi possível verificar que não houve resposta à terapia de terceira-linha em pacientes refratários ao tratamento de primeira-linha baseado em platina ${ }^{12}$. Este estudo permitiu concluir que topotecan e paclitaxel não apresentam resistência cruzada significativa, justificando o uso racional de topotecan como segunda-linha em câncer de ovário em pacientes com recaída após o uso de paclitaxel ${ }^{12}$.

Gordon et al. compararam topotecan e doxorrubicina lipossomal em um estudo americano de fase III, envolvendo 474 pacientes com câncer de ovário recidivado ${ }^{13}$. A taxa de resposta global foi de $17 \%$ e $19,7 \%$ para os grupos que receberam topotecan e doxorrubicina, respectivamente $(\mathrm{p}=0,39)$. Não houve diferença estatisticamente significativa para sobrevida livre de progressão $(\mathrm{p}=0,095)$ e sobrevida mediana $(\mathrm{p}=0,341)$. Analisando apenas as pacientes refratárias à platina, observou-se taxa de resposta global de 6,5\% para o grupo do topotecan e $12,3 \%$ para o grupo da doxorrubicina lipossomal $(\mathrm{p}=0,118)$. A sobrevida mediana livre de progressão foi de 9,1 semanas e 13,6 semanas para topotecan e doxorrubicina lipossomal, respectivamente $(\mathrm{p}=0,733)$, enquanto a sobrevida mediana global foi de 35,6 e 41,3 semanas, respectivamente $(\mathrm{p}=0,455)$. Já entre as pacientes sensíveis à platina, houve diferença estatisticamente significativa na sobrevida livre de progressão, a favor da doxorrubicina lipossomal sobre o grupo do topotecan, com mediana de 28,9 e 23,3 semanas, respectivamente $(\mathrm{p}=0,037)$. Também, foi notada diferença significativa em sobrevida global, favorecendo o uso da doxorrubicina lipossomal, com mediana de 108 semanas contra 71,1 semanas no grupo do topotecan $(\mathrm{p}=0,008)$. $\mathrm{Na}$ análise global, houve diferença significativa no perfil de toxicidade, predominando neutropenia no grupo do topotecan ( $77 \%$ com graus 3 e 4 ) e eritrodisestesia palmoplantar no grupo da doxorrubicina lipossomal (49\% dos pacientes), ambos com $\mathrm{p}<0,001^{13}$.

Topotecan foi utilizado como quimioterápico de escolha no Hospital de Câncer II, do Instituto Nacional de Câncer (INCA), para as pacientes com tumor epitelial de ovário refratário à platina. $\mathrm{O}$ presente estudo tem como objetivo primário descrever o tempo livre de progressão (TLP) dessas pacientes tratadas com topotecan no período de janeiro de 2003 a dezembro de 2005. Outros objetivos incluídos foram: descrever os sítios de progressão mais freqüentes nessas pacientes; descrever as taxas de resposta clínica, bioquímica e radiológica dessas pacientes ao tratamento com topotecan; descrever a sobrevida global (SG) da população estudada.

\section{PACIENTES E MÉTODOS}

\section{DESENHO DO ESTUDO}

Estudo de seguimento, retrospectivo e descritivo, realizado através da avaliação dos prontuários das pacientes, com diagnóstico de câncer epitelial de ovário refratário à platina, que receberam tratamento com topotecan no INCA no período de janeiro de 2003 a dezembro de 2005. A lista das pacientes foi obtida através dos registros do Sistema de Informaçóes Hospitalares (SIH) da unidade II do INCA. As informaçóes colhidas 
através da revisão de prontuários foram arquivadas em formulário padronizado. Entre as características das pacientes, foram descritas a idade, performance status ${ }^{14}$ (PS), estágio patológico de FIGO, grau e tipo histológico e tipo de cirurgia primária (quando realizada). Em relação aos tratamentos quimioterápicos, foram descritas as drogas utilizadas, número de ciclos, data dos ciclos, bem como a taxa de resposta clínica, bioquímica e resposta documentada a partir de métodos de imagem. As fichas foram colocadas em planilha eletrônica (Microsoft Excel 97 ${ }^{\mathrm{TM}}$ ) para análise estatística com o pacote estatístico SPSS 11.0 (SPSS Inc., Califórnia, EUA). O tempo livre de progressão e a sobrevida global foram determinados através da construção de curvas de Kaplan-Meier.

\section{CRITÉRIOS DE ELEGIBILIDADE}

Foram incluídas as pacientes tratadas com topotecan no Instituto Nacional de Câncer e com diagnóstico de tumor epitelial de ovário refratário à platina no período de janeiro de 2003 a dezembro de 2005. Foram consideradas refratárias à platina aquelas pacientes que apresentaram progressão da doença durante o tratamento com esquemas de quimioterapia baseada em platina, ou que tiveram intervalo livre de progressão menor do que seis meses após tratamento prévio com platinas. Foram excluídas da análise pacientes com tumor de ovário de subtipos não-epiteliais, pacientes, que não receberam tratamento com topotecan, e pacientes, que receberam topotecan por menos do que um ciclo prescrito (pelo menos três dias do tratamento).

\section{CONCEITOS}

O tempo, em meses, entre o primeiro dia do primeiro ciclo de topotecan até a documentação de progressão da doença, morte, ou interrupção do seguimento foi denominado TLP. A SG é aqui definida como o intervalo de tempo entre o primeiro dia do primeiro ciclo de topotecan até a data de óbito ou data da última visita ao hospital (serviço de emergência ou visita ambulatorial). Os pacientes ainda vivos foram censurados na data correspondente à última consulta no Instituto.

A avaliação de resposta radiológica realizada, após o último ciclo de topotecan, foi feita através de doença mensurável de forma unidimensional por imagem (radiografia simples, ultra-sonografia, tomografia computadorizada e ressonância nuclear magnética). Utilizou-se o termo resposta completa (RC) para documentar o desaparecimento de toda doença mensurável, na ausência de novas lesōes ${ }^{15}$. Resposta parcial (RP) foi definida como redução $\geq 30 \%$ na soma dos maiores diâmetros de todas as lesões tumorais ${ }^{15}$.
Progressão de doença (PD) foi documentada nas pacientes com aumento $\geq 20 \%$ na soma dos maiores diâmetros das lesões tumorais, reaparecimento de lesão, que havia desaparecido, e aparecimento de nova lesão ${ }^{15}$. Foram classificados como doença estável (DE) aquelas pacientes com redução tumoral, porém sem critérios suficientes para RP, bem como aquelas com aumento de volume tumoral, que não preencheram critérios para $\mathrm{PD}$, tendo como referência a menor soma dos maiores diâmetros desde o início do tratamento ${ }^{15}$.

Os níveis séricos de CA-125 foram utilizados como marcadores de resposta bioquímica. Seguindo os critérios de Rustin et al., a redução dos níveis séricos de CA-125, em 50\% ou mais em duas amostras, confirmada em uma quarta amostra, ou a redução de $75 \%$ ao longo de três amostras foram consideradas como critério bioquímico de resposta ${ }^{16}$. Progressão de doença por critérios bioquímicos ficou definida pela demonstração de aumento do CA-125 em três amostras com piora concomitante dos sintomas ou quatro amostras sem piora de sintomas ${ }^{16}$.

Entre os critérios clínicos de avaliação de resposta, foram considerados o PS (variando de zero a quatro) ${ }^{14}$, avaliação subjetiva do controle da dor através de escala visual analógica ${ }^{17}$ (convertida a escala numérica, variando de zero a dez, para fins de registro) e da necessidade do uso de analgésicos, avaliação da freqüência da realização de paracenteses para alívio de sintomas relacionados à ascite, análise unidimensional de lesões clinicamente mensuráveis, assim como continuidade do seguimento. Aumento $\geq 20 \%$ no diâmetro de lesōes mensuráveis, surgimento de novas lesôes ou novos sintomas, piora da dor segundo escala numérica, uso mais freqüente de analgésicos, piora do PS, maior necessidade de paracenteses, assim como perda de avaliação por interrupção do seguimento ou morte foram considerados critérios clínicos de progressão de doença. Redução da intensidade da dor, uso menos freqüente de analgésicos, melhora do PS, menor necessidade de paracenteses, redução $\geq 30 \%$ no diâmetro de lesões clinicamente mensuráveis ou desaparecimento completo sem surgimento de novas lesōes foram considerados critérios de resposta clínica. Pacientes sem critérios de resposta ou progressão foram classificados como tendo obtido doença clínica estável.

\section{ASPECTOS ÉTICOS ENVOLVIDOS}

O presente protocolo foi aprovado pelo Comitê de Ética em Pesquisa (CEP) do INCA. Trata-se de um estudo descritivo, no qual são analisados dados obtidos a partir de anotações em prontuários médicos, exame 
clínico e exames laboratoriais, que fazem parte da assistência habitual prestada pela equipe multiprofissional do setor. Não houve nenhuma forma de intervenção diagnóstica, terapêutica ou de qualquer natureza, motivo pelo qual não se utilizou consentimento informado. Todos os aspectos éticos envolvidos encontram-se de acordo com a resolução CNS 196/96 (DO. No 201, 16 de outubro de 1996) e suas complementares.

\section{RESULTADOS}

Através de uma lista de pacientes obtida pelos registros do SIH da unidade II do INCA, encontrou-se um total de 36 pacientes. Dessas pacientes, quatro não utilizaram topotecan, pois apresentaram progressão de doença antes do início do tratamento e foram consideradas inelegíveis. Uma paciente apresentava um tumor neuroectodérmico de vulva e não tumor de ovário. Essas cinco pacientes foram excluídas do estudo que, para avaliação dos objetivos previstos, incluiu trinta e uma pacientes, que receberam pelo menos três dias do tratamento para tumor de ovário refratário à platina.

A idade mediana das pacientes foi de 53 anos, variando de 42 a 77 . Em relação ao estadiamento (FIGO), duas pacientes tinham estágio inicial I $(6,5 \%)$, 25 pacientes tinham estágio III $(80,6 \%)$ e quatro pacientes tinham estágio IV $(12,9 \%)$. O subtipo histológico mais encontrado foi o seroso papilífero, descrito no tumor de 18 pacientes $(58,1 \%)$. Outros subtipos encontrados foram: mucinoso e sero-mucinoso em duas pacientes cada ( $6,5 \%$ cada), pouco diferenciado em sete pacientes $(22,6 \%)$ e indeterminado em duas pacientes $(6,5 \%)$. Das 26 pacientes com descrição do grau de diferenciação tumoral, três tinham tumor grau $1(11,5 \%), 11$ tinham tumor grau $2(42,3 \%)$ e 12 tinham tumor grau $3(46,2 \%)$. Todas as 31 pacientes haviam sido submetidas à citorredução primária, sendo conseguido citorredução ótima em apenas quatro pacientes (12,9\%), subótima em seis pacientes $(19,4 \%)$, doença residual em $20(64,5 \%)$ e em uma paciente o grau de citorredução não foi especificado $(3,2 \%)$. Dezenove pacientes se submeteram a outra intervenção com intenção de citorredução e, avaliando todas as operações, foi atingida citorredução ótima em nove pacientes (29\%), subótima em seis $(19,4 \%)$ e doença residual em 16 (51,6\%). Ao início do tratamento com topotecan, três pacientes tinham PS de $0(9,7 \%), 20$ tinham PS $1(64,5 \%)$ e oito tinham PS $2(25,8 \%)$. Cinco das pacientes receberam radioterapia, duas para paliação de doença com recaída em cúpula vaginal, duas para paliação de massa pélvica e uma paciente para paliação de nódulos subcutâneos em parede abdominal. As pacientes haviam recebido de três a 21 ciclos de quimioterapia baseada em platina, com mediana de oito ciclos, incluindo tratamento adjuvante e paliativo. A progressão após o último ciclo com platina ocorreu em até seis meses, com mediana de um mês. As pacientes haviam recebido, no total, de duas a quatro classes de quimioterápicos previamente ao uso de topotecan, com mediana de dois. Os dados referentes às características de base estão resumidos na tabela 1 .

Com seguimento mediano de 12 meses (3-36), entre as 31 pacientes descritas, 27 tinham apresentado progressão da doença após o tratamento com topotecan $(87,8 \%)$ e 19 haviam evoluído para óbito

Tabela 1. Características de base dos pacientes tratados com topotecan $(n=31)$

\begin{tabular}{l|c}
\hline \multicolumn{1}{c|}{ Características } & Número de pacientes (\%) \\
\hline Idade mediana (anos) & $53(42-77)$ \\
PS & \\
0 & $3(9,7 \%)$ \\
1 & $20(64,5 \%)$ \\
2 & $8(25,8 \%)$ \\
3 & 0 \\
Estágio (FIGO) & \\
I & $2(6,5 \%)$ \\
II & 0 \\
III & $25(80,6 \%)$ \\
IV & $4(12,9 \%)$ \\
Histologia & $18(58,1 \%)$ \\
Seroso papilífero & $2(6,5 \%)$ \\
Sero-mucinoso & $2(6,5 \%)$ \\
Mucinoso & $7(22,6 \%)$ \\
Pouco diferenciado & $2(6,5 \%)$ \\
Indeterminado & \\
Grau & $3(9,7 \%)$ \\
1 & $11(35,5 \%)$ \\
2 & $12(38,7 \%)$ \\
3 & $5(16,1 \%)$ \\
Níño relatado & $4(12,9 \%)$ \\
Orótima & $6(19,4 \%)$ \\
Supós pótima & $20(64,5 \%)$ \\
Doença residual & $1(3,2 \%)$ \\
Não especificada & $5(16,1 \%)$ \\
\hline
\end{tabular}

Abreviação: PS = performance status. 
$(61,2 \%)$. O tempo mediano livre de progressão foi de quatro meses (IC 95\%; 2,1-5,8) e a mediana estimada de sobrevida global foi de 14 meses (IC 95\%; 5,1-22,8). As curvas de Kaplan-Meier de tempo livre de progressão e sobrevida global estão expostas como figuras 1 e 2 , respectivamente.

Em relação ao tratamento com topotecan, as pacientes receberam de dois a dez ciclos de quimioterapia, com mediana de cinco ciclos. Quatorze pacientes completaram pelo menos os seis ciclos propostos $(45,2 \%)$. Entre as 17 pacientes, que não completaram esses ciclos, o principal motivo de abandono foi progressão de doença, o que ocorreu em 14 pacientes $(45,2 \%)$. Toxicidade foi a causa de abandono em três $(9,7 \%)$. A dose mediana utilizada por ciclo foi de 6,36 (+-1,09 desvio padrão) $\mathrm{mg} / \mathrm{m}^{2} /$ ciclo.

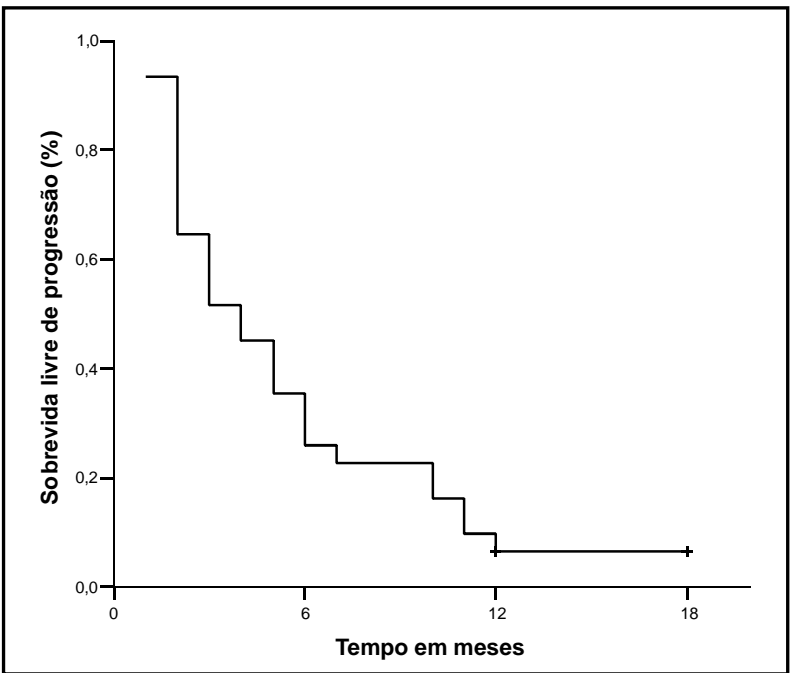

Figura 1. Curva de Kaplan-Meier de sobrevida livre de progressão

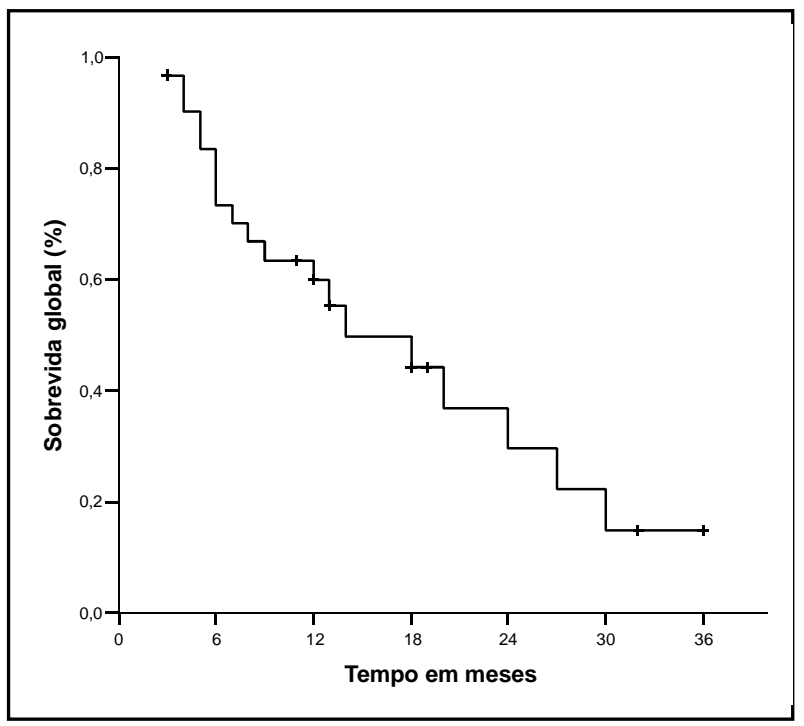

Figura 2. Curva de Kaplan-Meier de sobrevida global
Sete pacientes obtiveram critérios de resposta clínica com o tratamento $(22,6 \%)$, seis pacientes obtiveram doença clínica estável (19,3\%) e 18 pacientes apresentaram progressão clínica de doença $(58,1 \%)$. Durante os seis ciclos de topotecan, oito pacientes tiveram resposta bioquímica $(25,8 \%), 15$ pacientes tiveram critérios para progressão bioquímica $(48,4 \%)$ e oito pacientes mantiveram níveis estáveis de CA-125 $(25,8 \%)$. Vinte e cinco pacientes tiveram reavaliação de resposta por métodos de imagem, sendo encontrada resposta parcial em cinco pacientes $(20 \%)$, progressão de doença em 11 pacientes (44\%) e doença estável em nove pacientes $(36 \%)$. Esses valores são também apresentados na tabela 2 .

Tabela 2. Resposta ao tratamento com topotecan

\begin{tabular}{l|c|c}
\hline \multicolumn{1}{c|}{ Resposta } & $\begin{array}{c}\text { Número de } \\
\text { pacientes }\end{array}$ & $\begin{array}{c}\text { Porcentagem } \\
\text { (\%) }\end{array}$ \\
\hline $\begin{array}{l}\text { Resposta } \\
\text { radiológica } \\
\text { (n=25) }\end{array}$ & & \\
$\begin{array}{l}\text { Resposta parcial } \\
\text { Doença estável }\end{array}$ & 5 & 20 \\
$\begin{array}{l}\text { Progressão de } \\
\text { doença }\end{array}$ & 11 & 44 \\
$\begin{array}{l}\text { Resposta } \\
\text { bioquímica } \\
\text { (n=31) }\end{array}$ & 9 & 36 \\
$\begin{array}{l}\text { Resposta parcial } \\
\text { Doença estável } \\
\text { Progressão de } \\
\text { doença }\end{array}$ & 8 & \\
Resposta clínica \\
(n=31)
\end{tabular}

Entre as 27 pacientes, que tiveram progressão de doença até o momento da análise, a progressão clínica foi documentada em 25 pacientes (92,5\%), bioquímica em 21 pacientes $(77,7 \%)$ e por imagem em $16(59,2 \%)$. A progressão locorregional de doença foi a mais freqüente, documentada em 22 pacientes $(81,5 \%)$. Quatro pacientes tiveram progressão a distância (14,8\%) e uma paciente teve progressão com sítio não relatado $(3,7 \%)$. O primeiro exame a demonstrar progressão foi a tomografia computadorizada em 14 pacientes $(51,8 \%)$, ultra-som em cinco $(18,5 \%)$ e ressonância nuclear magnética em uma paciente (3,7\%). Após progressão 
de doença, a principal conduta foi a realização de nova linha de quimioterapia, decisão tomada para 22 pacientes $(81,4 \%)$. Dentre as quimioterapias utilizadas, estavam doxorrubicina lipossomal, gencitabina, etoposídeo oral, cisplatina e carboplatina.

\section{DISCUSSÃO}

Como estudo descritivo, o presente levantamento se limita a verificar os desfechos e as características relacionadas às pacientes e ao tratamento ${ }^{18}$. $\mathrm{O}$ desenho do estudo não inclui, portanto, análise de associações como a hipótese de que o uso de topotecan aumente o TLP ou a SG na população estudada, tendo em vista a ausência de grupo controle ${ }^{18}$. Além disso, a coleta de informaçóes sobre os casos foi realizada retrospectivamente, através de revisão de prontuários. Essa forma de coleta é sabidamente limitada e pode estar sujeita a diversos tipos de erros sistemáticos ${ }^{18}$. São comumente encontrados dados incompletos em prontuário, bem como existe heterogeneidade nas condutas tomadas pelos médicos, que assistiram os pacientes, incluindo a dose utilizada do tratamento, exames solicitados para o acompanhamento das pacientes, freqüência dos exames, modificações no esquema de tratamento devido a efeitos tóxicos e conduta após progressão de doença. Por outro lado, o presente estudo reflete a prática atual em nosso serviço e tem a vantagem de gerar informaçóes sobre os resultados obtidos, considerando peculiaridades encontradas em nossa prática diária.

No período de janeiro de 2003 a dezembro de 2005, 31 pacientes receberam tratamento com topotecan com intuito paliativo neste tipo de situação. Com seguimento mediano de 12 meses, foi observado TLP de quatro meses e sobrevida global mediana de 14 meses. O TLP descrito é aparentemente inferior aos dados da literatura, principalmente quando se avaliam os estudos de fase II, que citam intervalo livre de progressão, variando de quatro a 13 meses. Já em avaliaçôes de fase III, foi descrito tempo livre de progressão, variando de nove a 23 semanas. Algumas características das pacientes incluídas no presente trabalho, entretanto, sugerem pior prognóstico e devem ser consideradas durante a análise desses dados. Todas as pacientes aqui avaliadas foram consideradas refratárias à platina, com tempo mediano após o último ciclo de platina de apenas um mês, variando de zero a seis meses. Os estudos citados com freqüência descrevem análises conjuntas de pacientes refratárias com pacientes sensíveis à platina e demonstram tempo mediano de até sete meses após último ciclo de quimioterapia prévia ao uso de topotecan. Além disso,
$88,5 \%$ da nossa amostra com descrição do grau de diferenciação neoplásica apresentam tumores pouco ou moderadamente diferenciados e um quarto das pacientes aqui incluídas tinha PS 2.

A sobrevida mediana de 14 meses se assemelha àquela citada na literatura. A SG variou de 47 semanas até 20 meses em estudos de fase II e de 35 a 61 semanas em estudos de fase III. O presente estudo traz como dado de interesse a conduta seguida após progressão de doença com uso de topotecan. A grande maioria das pacientes $(81,4 \%)$ recebeu outras linhas de quimioterapia, incluindo participação em protocolos de pesquisa. Esse tipo de informação é pouco citado na literatura e pode ter influência sobre a taxa de sobrevida global.

Com relação à taxa de resposta, o presente estudo encontrou resposta bioquímica de $25,8 \%$, radiológica de $20 \%$ e clínica em $22,6 \%$ das pacientes. Esses resultados são compatíveis com aqueles da literatura, com citações de taxa de resposta variando de $13,7 \%$ a $32,6 \%$ em estudos de fase II. Entre os estudos de fase III, são citados valores de 6,5\% em análise isolada de pacientes refratárias à platina até taxa de resposta de $20,5 \%$ em análise, englobando pacientes refratárias e sensíveis à platina.

Os dados do presente estudo evidenciam o benefício do tratamento com topotecan na população estudada, com taxa de resposta, tempo livre de progressão e sobrevida global semelhantes aos escritos na literatura.

\section{REFERÊNCIAS}

1. Jemal A, Siegel R, Ward E, Murray T, Xu J, Thun MJ. Cancer statistics 2007. CA Cancer J Clin. 2007;57(1):43-66.

2. Instituto Nacional de Câncer. Câncer no Brasil: dados dos registros de base populacional. Rio de Janeiro (RJ): INCA; 2003. vol. 3.

3. Salom E, Almeida Z, Mirhashemi R. Management of recurrent ovarian cancer: evidence-based decision. Curr Opin Oncol. 2002;14(5):519-27.

4. Karlan BY, Markman MA, Eifel PJ. Ovarian cancer, peritoneal carcinoma and pallopian tube carcinoma. In: De Vita Jr, Vincent T, Hellman S, Rosenberg SA. Cancer: principles and practice of oncology. 7th ed. Philadelphia: Lippincott Williams \& Wilkins; 2005. p. 1364-97.

5. Markman M, Markman J, Webster K, Zanotti K, Kulp B, Peterson G, et al. Duration of response to second-line, platinum-based chemotherapy for ovarian cancer: implications for patient management and clinical trial design. J Clin Oncol. 2004;22(15):3120-5.

6. Creemers GJ, Bolis G, Gore M, Scarfone G, Lacave AJ, Guastalla JP, et al. Topotecan, an active drug in the second-line treatment of epithelial ovarian cancer: results of a large European phase II study. J Clin Oncol. 1996;14(12):3053-5. 
7. Kudelka AP, Tresukosol D, Edwards CL, Freedman RS, Levenback C, Chantarawiroj P, et al. Phase II study of intravenous topotecan as a 5-day infusion for refractory epithelial ovarian carcinoma. J Clin Oncol. 1996;14(5):1552-7.

8. Swisher EM, Mutch DG, Rader JS, Elbendary A, Herzog TJ. Topotecan in platinum and paclitaxe-resistant ovarian cancer. Gynecol Oncol. 1997;66(3):480-6.

9. Bookman MA, Malmstrom H, Bolis G, Gordon A, Lissoni A, Krebs JB, et al. Topotecan for the treatment of advanced epithelial ovarian cancer: an open-label phase II study in patients treated arfter prior chemotherapy that contained cisplatin or carboplatin and paclitaxel. J Clin Oncol. 1998;16(10):3345-52.

10. McGuire WP, Blessing JA, Bookman MA, Lentz SS, Dunton CJ. Topotecan has substantial antitumor activity as first-line salvage therapy in platinum-sensitive epithelial ovarian carcinoma; a gynecologic oncology group study. J Clin Oncol. 2000;18(5):1062-7.

11. Huinink WTB, Gore M, Carmichael J, Gordon A, Malfetano J, Hudson I, et al. Topotecan versus paclitaxel for the treatment of recurrent epithelial ovarian cancer. J Clin Oncol. 1997;15(6):2183-93.

12. Gore M, Huinink WTB, Carmichael J, Gordon A, Davidson $\mathrm{N}$, Coleman R, et al. Clinical evidence for topotecanpaclitaxel non-cross-resistance in ovarian cancer. J Clin Oncol. 2001;19(7):1893-900.
13. Gordon NA, Fleagle JT, Guthrie D, Parkin DE, Gore ME, Lacave AJ. Recurrent epithelial ovarian carcinoma: a randomized phase III study of pegylated liposomal doxorubicin versus topotecan. J Clin Oncol. 2001;19(14):3312-22.

14. Oken MM, Creech RH, Tormey DC, Horton J, Davis TE, MacFadden ET, et al. Toxicity and response of the Eastern Cooperative Oncology Group. Am J Clin Oncol. 1982;5(6):649-55.

15. Therasse P, Arbuck SG, Eisenhauer EA, Wanders J, Kaplan RS, Rubinstein L, et al. New guidelines to evaluate the response to treatment in solid tumors. J Natl Cancer Inst. 2000;92(3):205-16.

16. Rustin GJS, Nelstrop ARE, Crawford M, Lederman J, Lambert HE, Coleman LR, et al. Phase II trial of oral altretamine for relapsed ovarian carcinoma: evaluation of defining response by serum CA-125. J Clin Oncol. 1997;15(1):172-6.

17. Caraceni A, Cherny N, Faisinger R, Kaasa S, Poulain P, Radbruch L, et al. Pain measurement tools and methods in clinical research in palliative care: recommendations of an expert working group of the European Association of Palliative Care. J Pain Symptom Manage. 2002;23(3):239-55.

18. Rothman KJ, Greenland S. Types of epidemiologic studies. In: Rothman KJ, Greenland S. Modern epidemiology. 2nd ed. Philadelphia: Lippincott Williams \& Wilkins; 1998. p. 67-78.

\begin{abstract}
Introduction: Ovarian cancer is the leading cause of death from gynecological neoplasms in the United States. Despite advances in initial therapy, most patients eventually develop progressive disease and require further treatment. Topotecan, an active drug used for the treatment of patients with ovarian disease, has been used at the Brazilian National Cancer Institute. The aim of the present study was to describe such clinical cases. Methods: This was a retrospective and descriptive study with review of files of patients with recurrent platinum-refractory ovarian cancer. Patients had received topotecan as second-line therapy from January 2003 to December 2005 at the Brazilian National Cancer Institute. Results: Thirty one patients were eligible. After a median follow-up of twelve months (range 3-36), progression-free survival was four months (95\%CI: 2.1-5.8) and overall survival was 14 months (95\%CI: 5.1-22.8). The most common form of progression was locoregional, found in 22 patients $(81.5 \%)$. Clinical response was achieved in $45.2 \%$ of patients, while the biochemical and radiological response rates were $25.8 \%$ and $20.0 \%$, respectively. Conclusion: Topotecan proved to be an effective therapy in this group of patients. The response rate, progression-free survival, and overall survival were comparable to those observed in randomized trials.
\end{abstract}

Key words: Ovarian neoplasms; Drug therapy; Topotecan 